\title{
417 - Comparison of prevalence and disturbance of neuropsychic symptoms between institution-dwelling and community-dwelling patients with dementia
}

\author{
Xuejiao Lu, Jialan Wu, Rui Ye, Xiaoyan Liao*
}

\begin{abstract}
Objectives To explore differences in prevalence and disturbance of behavioral and psychological symptoms of dementia (BPSD) between institution-dwelling and community-dwelling patients with dementia.
\end{abstract}

Methods A cross-sectional survey was conducted. Institution-dwelling ( $n=112)$ and community-dwelling patients with dementia $(n=157)$ were enrolled from a nursing home and a tertiary psychiatric hospital located in Guangzhou, China, from March 2019 to September 2019. Neuropsychiatric Inventory (NPI) was used to assess the prevalence and disturbance of BPSDs.

Results The community-dwelling patients were older, and having more antianxiety, anticonvulsant, antidepression, anti-dementia medication use than institution-dwelling patients, while institution-dwelling patients used more antipsychotics than community-dwelling patients. The prevalence of hallucinations $\left(\chi^{2}=8.55, P=0.004\right)$, agitation $\left(\chi^{2}=5.38, P=0.026\right)$, depression $\left(\chi^{2}=22.78, P<0.001\right)$, anxiety $\left(\chi^{2}=\right.$ 47.17, $P<0.001)$, aberrant motor behavior $\left(\chi^{2}=4.72, P=0.034\right)$, and sleep $\left(\chi^{2}=4.16, P=0.047\right)$ was higher in community-dwelling patients than that in institution-dwelling patients. Moreover, the frequency, severity, and disturbance of almost all of the BPSDs were higher in community-dwelling patients than those in institution-dwelling patients, except for euphoria. High Odds of having hallucination (OR 2.48, 95\% Cl 1.37-4.49), anxiety (OR 7.36, 95\% Cl 3.82-14.17), sleep disorder (OR 1.77, $95 \% \mathrm{Cl}$ 1.02-3.10) was observed in community-dwelling patients. Severe dementia was another risk factor for having hallucination (OR 5.48, 95\% Cl 2.25-13.38), anxiety (OR 2.73, 95\% Cl 1.22-6.12), sleep disorder (OR 2.61, 95\% Cl 1.21-5.64), apathy (OR 3.03, 95\% Cl 1.49-6.19), and irritability (OR 2.6, 95\% Cl 1.24-5.51). Community-dwelling and severe dementia were two main risk factors for higher NPI scores (adjusted $R^{2}=0.30$ ), and higher disturbance of anxiety (adjusted $R^{2}=0.21$ ), sleep disorders (adjusted $R^{2}=$ 0.15 ), hallucination (adjusted $R^{2}=0.12$ ) and irritability (adjusted $R^{2}=0.11$ ).

Conclusion The prevalence and disturbance of BPSDs were higher in community-dwelling patients with dementia than institution-dwelling patients. Community-dwelling and severe dementia were two main risk factors for higher prevalence and disturbance of BPSDs, especially for anxiety, sleep disorders, and hallucination. Providing community-based BPSDs coping skill training and support for informal caregivers are needed.

Keywords: Community, Dementia, Long-term care, Informal caregiver, Neuropsychiatric symptoms

\section{8 - Long-term Effect of Transcranial Direct Current Stimulation (tDCS) in Alzheimer's} Dementia

Carol Sheei-Meei Wang ${ }^{1,2,3 *}$, Kuo-Sheng Cheng ${ }^{2}$, Chia-Hung Tang ${ }^{1}$, Nien-Tsen Hou ${ }^{4}$, Pei-Fang Chien ${ }^{1}$, Ying-Che Huang ${ }^{4}$

\footnotetext{
${ }^{1}$ Department of Psychiatry, Tainan Hospital, Ministry of Health and Welfare, Tainan City, Taiwan

${ }^{2}$ Department of BioMedical Engineering, National Cheng Kung University, Tainan City, Taiwan

${ }^{3}$ Department of Psychiatry, National Cheng Kung University Hospital, Tainan City, Taiwan

${ }^{4}$ Department of Neurology, Tainan Hospital, Ministry of Health and Welfare, Tainan City, Taiwan
} 


\begin{abstract}
:
Introduction: To explore and develop effective treatments is crucial for patients with Alzheimer's dementia (AD). In pathology, the amyloid deposits of $A D$ result in disruption of the balance between long-term potentiation (LTP) and long-term depression (LTD) of neuronal cells and synaptic plasticity. Transcranial direct current stimulation (tDCS) has been proposed to affect long-term synaptic plasticity through LTP and LTD, thereby improving cognitive ability. Although an increasing number of studies have been concluded a positive therapeutic effect on cognition in AD, tDCS studies to date are limited on exploring the duration of its efficacy. In this pilot study, we investigate the effects of tDCS in AD and verify its extending beneficial effects for 3 months follow-up period after the end of stimulation.
\end{abstract}

Method: 34 AD participants aged 55-90 years (mean age 75.9 (66-86)) were included in a double-blind, randomized, sham-controlled crossover study. All participants were randomly assigned to receive 10 consecutive daily sessions of active tDCS (or sham) and switched groups 3 months later. The anodal electrode was on the left dorsal lateral prefrontal cortex and the cathodal electrode was on the right supraorbital area. In each active session, we applied a current intensity of $2 \mathrm{~mA}$ and an electrode size of $25 \mathrm{~cm}^{2}$ for $30 \mathrm{~min}$ in the active group. All subjects received a series of neuropsychological assessments including CDR, MMSE, CASI and WCST at baseline and in 2 weeks, 4 weeks, and 12 weeks post-tDCS (or sham) 10 sessions. Chi-square tests, Wilcoxon signed rank tests and Mann-Whitney $U$ tests were used to assess the differences in participant demographic characteristics and to compare the differences of test scores between groups.

Results: The active tDCS group showed significant improvements on CASI total scores from baseline to 2-weeks, 1-month and 3-months after active stimulations, though the improvement declined over time. There are also different presentations in total correct items, conceptual level responses, failure to maintain sets of WCST between active tDCS and sham groups. There is no difference in MMSE, CASI and WCST scores in the sham groups.

Conclusion: These results suggest a long term-beneficial effects of tDCS in AD.

Key words: Transcranial Direct Current Stimulation, Alzheimer's dementia, cognitive function, Wisconsin Card Sorting Test

\title{
419 - Effect of Transcranial Direct Current Stimulation (tDCS) in Dementia with Lewy Bodies Carol Sheei-Meei Wang ${ }^{1,2,3 *}$, Kuo-Sheng Cheng ${ }^{2}$, Chia-Hung Tang ${ }^{1}$, Nien-Tsen Hou ${ }^{4}$, Pei-Fang Chien ${ }^{1}$, Ying-Che Huang ${ }^{4}$
}

${ }^{1}$ Department of Psychiatry, Tainan Hospital, Ministry of Health and Welfare, Tainan City, Taiwan

${ }^{2}$ Department of BioMedical Engineering, National Cheng Kung University, Tainan City, Taiwan

${ }^{3}$ Department of Psychiatry, National Cheng Kung University Hospital, Tainan City, Taiwan

${ }^{4}$ Department of Neurology, Tainan Hospital, Ministry of Health and Welfare, Tainan City, Taiwan

\begin{abstract}
:
Introduction: Dementia with Lewy Bodies (DLB), this second most common form of degenerative dementia, presents more functional disability, more potentially fatal complication, more impaired quality of life than Alzheimer's dementia. There is no FDA-proved medication can slow, stop or improve the progression of cognitive declines in DLB. Identifying effective treatments is a critical issue for DLB. In neuropathology, extracelluar $\alpha$-syn oligomers interfere with the expression of long-term potentiation, and influence memory and learning. Transcranial direct current stimulation (tDCS) has been proposed to affect long-term synaptic plasticity through LTP and LTD, thereby improving
\end{abstract}

